ISSN: 2503-3697, e-ISSN: 2541-2590

Vol. 3, No. 2, July 2018

http://journals.ums.ac.id/index.php/jramathedu

\title{
Acknowledgement for Reviewers
}

The Editors of JRAMathEdu would like to express their sincere gratitude to the following people, who reviewed manuscripts for Volume 32018.

Toh Tin Lam, National Institute of Education, Nanyang Technological University, SINGAPORE (SCOPUS ID: 16403102700)

Sharyn Livy, Faculty of Education, Monash University, AUSTRALIA (SCOPUS ID: $55887811700)$

Abdur Rahman As'ari, Department of Mathematics Education, Universitas Negeri Malang, INDONESIA (SCOPUS ID: 57201667466)

Akhsanul In'am, Department of Mathematics Education, Universitas Muhammadiyah Malang, INDONESIA (SCOPUS ID: 55639769600)

Hongki Julie, Department of Mathematics Education, Universitas Sanata Dharma, Yogyakarta, INDONESIA (SCOPUS ID: 57190939380)

Nining Setyaningsih, Department of Mathematics Education, Universitas Muhammadiyah Surakarta, INDONESIA (SCOPUS ID: 57194533654)

Janu Arlinwibowo, Department of Mathematics Education, Universitas Ahmad Dahlan, Yogyakarta, INDONESIA (SCOPUS ID: 57194702146)

Fariz Setiawan, Department of Mathematics Education, Universitas Ahmad Dahlan, Yogyakarta, INDONESIA (SCOPUS ID: 57200658876)

Afit Istiandaru, Department of Mathematics Education, Universitas Ahmad Dahlan, Yogyakarta, INDONESIA (SCOPUS ID: 57200659808)

Rasiman, Department of Mathematics Education, Universitas PGRI, Semarang, INDONESIA (SINTA ID: 0018025601 )

Sunandar, Department of Mathematics Education, Universitas PGRI Semarang, INDONESIA ( SINTA ID: 6022217)

Budi Murtiyasa, Department of Mathematics Education, Universitas Muhammadiyah Surakarta, INDONESIA (SINTA ID: 5978134)

Idris Harta, Department of Mathematics Education, Universitas Muhammadiyah Surakarta, INDONESIA (SINTA ID: 6191423)

Sutama, Department of Mathematics Education, Universitas Muhammadiyah Surakarta, INDONESIA (SINTA ID: 5985034)

Wahid Yunianto, SEAMEO QITEP in Mathematics, INDONESIA 